\title{
Molecular characterization and distribution of Cryptosporidium spp., Giardia duodenalis, and Enterocytozoon bieneusi from yaks in Tibet, China
}

Yayun Wu ${ }^{1,2}$, Yankai Chang ${ }^{1,2}$, Xiangqian Zhang ${ }^{1,2}$, Yuancai Chen ${ }^{1,2}$, Dongfang Li $\mathrm{Li}^{1,2}$, Lu Wang ${ }^{1,2}$, Shuangjian Zheng ${ }^{1,2}$, Rongjun Wang ${ }^{1,2}$, Sumei Zhang ${ }^{1,2}$, Fuchun Jian ${ }^{1,2}$, Changshen Ning ${ }^{1,2}$, Jiakui Li ${ }^{3,4}$ and Longxian Zhang ${ }^{1,2^{*}}$ (D)

\begin{abstract}
Background: With worldwide distribution and importance for veterinary medicine, Cryptosporidium spp., Giardia duodenalis, and Enterocytozoon bieneusi have been found in a wide variety of vertebrate hosts. At present, few available molecular data can be used to understand the features of genetic diversity of these pathogens in areas without or less intensive farming. Dominated by grazing, Tibet is a separate geographic unit in China and yaks are in frequent contact with local herdsmen and necessary for their daily life. Therefore, to investigate the distribution of these pathogens in yaks of Tibet, 577 fecal specimens were screened using nested PCR for the presence and genotypes of the three intestinal pathogens.

Results: The overall prevalence of Cryptosporidium spp., G. duodenalis, and E. bieneusi were 1.4\% (8/577), 1.7\% $(10 / 577)$, and 5.0\% (29/577), respectively. Cryptosporidium andersoni $(n=7)$ and Cryptosporidium bovis $(n=1)$ were detected by sequence analysis of the SSU rRNA gene. Genotyping at the SSU rRNA and triosephosphate isomerase genes suggested that all $G$. duodenalis positive specimens belonged to assemblage E. Sequence analysis of the internal transcribed spacer gene identified six known E. bieneusi genotypes: BEB4 $(n=11), I(n=6), \mathrm{D}(n=5)$,

$\mathrm{J}(n=2), \mathrm{CHC} 8(n=1)$, and BEB6 $(n=1)$. One subtype (A5,A4,A2,A1) for C. andersoni and three multilocus genotypes for E. bieneusi were identified by multilocus sequence typing.

Conclusions: We report for the first time the status of three enteric pathogens infection simultaneously for grazing yaks in Tibet. Yaks in our study are likely to impose a low zoonotic risk for humans. The molecular epidemiology data add to our knowledge of the characteristics of distribution and transmission for these pathogens in Tibet and their zoonotic potential and public health significance.
\end{abstract}

Keywords: Cryptosporidium spp., Giardia duodenalis, Enterocytozoon bieneusi, Zoonotic, Grazing, Tibet, Yaks

\footnotetext{
* Correspondence: zhanglongxian8999@foxmail.com;

zhanglx8999@gmail.com

${ }^{1}$ College of Animal Science and Veterinary Medicine, Henan Agricultural

University, Zhengzhou 450002, Henan Province, China

${ }^{2}$ International Joint Research Laboratory for Zoonotic Diseases of Henan,

Zhengzhou 450002, Henan Province, China

Full list of author information is available at the end of the article
}

(c) The Author(s). 2019 Open Access This article is distributed under the terms of the Creative Commons Attribution 4.0 International License (http://creativecommons.org/licenses/by/4.0/), which permits unrestricted use, distribution, and reproduction in any medium, provided you give appropriate credit to the original author(s) and the source, provide a link to the Creative Commons license, and indicate if changes were made. The Creative Commons Public Domain Dedication waiver (http://creativecommons.org/publicdomain/zero/1.0/) applies to the data made available in this article, unless otherwise stated. 


\section{Background}

As the commonly considered causes of human cryptosporidiosis, giardiosis, and microsporidiosis, Cryptosporidium spp., Giardia duodenalis, and Enterocytozoon bieneusi have been found in a wide variety of vertebrate hosts. These pathogens can present several typical clinical symptoms, varying from asymptomatic infection to acute or chronic diarrhea. Human infections are acquired by several transmission routes, such as direct contact with infected persons (anthroponotic transmission) or animals (zoonotic transmission) or ingestion of contaminated water or food [1-4]. Additionally, livestock have been identified as a source of some outbreaks of human cryptosporidiosis, giardiosis, and microsporidiosis [5-7]. The One Health concept recognizes that the health of people is connected to the health of animals and the environment and emphasizes the importance to study the distribution and transmission pathways of pathogens (http://www.cdc.gov/onehealth/).

Bovines have been identified as the mammals where Cryptosporidium infection is most universally found, and bovine cryptosporidiosis is predominantly attributed to Cryptosporidium parvum, C. bovis, C. andersoni and Cryptosporidium ryanae [8, 9]. From the limited studies on yaks, at least nine Cryptosporidium species and two genotypes have been described in yaks, including C. parvum, Cryptosporidium hominis, C. bovis, C. andersoni, C. ryanae, Cryptosporidium ubiquitum, Cryptosporidium xiaoi, Cryptosporidium struthionis, Cryptosporidium canis, Cryptosporidium occultus, C. ryanae cattle type, and $C$. ryanae buffalo type [8-10]. Currently, in terms of genetic variation, molecular biological analyses have shown that G. duodenalis has at least eight genotypes or assemblages (A-H), with A and B mainly infecting humans and animals and $\mathrm{C}$ to $\mathrm{H}$ being specifically restricted to nonhuman host species $[3,11]$. Assemblage $E$ is the most commonly reported in yaks, followed by assemblages $\mathrm{A}$ and $\mathrm{B}$ [12]. $E$. bieneusi has been genotyped into at least nine phylogenetic groups; zoonotic group 1 (also called the humanpathogenic group) includes almost all E. bieneusi genotypes from humans and some from animals, whereas groups 2-9 are generally considered host-adapted groups, suggesting no major public health significance [13, 14]. Among 13 genotypes detected in yaks from the three studies available, genotypes BEB4 and J were reported to be the dominant $E$. bieneusi genotypes in yaks, which were clustered into group 2. Additionally, genotypes CHN11, CHN12, CHN14, and WCY1 belonged to group 1 and the remainder belonged to group 2 [15-17].

Unlike that in other provinces and municipalities of China, the breeding industry is still dominated by grazing in Tibet, and free-range yaks and other abundant livestock are raised mostly on natural pastures and mountains. In addition to less Concentrated Animal Feeding Operation
(CAFO), Tibet is a separate geographic unit with the highest average elevation on earth. Approximately $90 \%$ of the world's yak population is distributed on the Qinghai-Tibet Plateau of China, with 4.9 million in Qinghai, 3.9 million in Tibet, 3.1 million in Sichuan, 0.88 million in Gansu, 0.17 million in Xinjiang, and 0.05 million in Yunnan [18]. As the representative livestock of Tibet and known as the "boat of the plateau", yaks are in frequent contact with local herdsmen and necessary for their daily life. Fresh fecal material from yaks is dried for cooking fuel, which increases the possibility of herder infection by Cryptosporidium, G. duodenalis, and E. bieneusi. Prior to our work, there was only an epidemiological study about Cryptosporidium and G. duodenalis in yaks from Damxung County in Tibet, in which no G. duodenalis infection was found [19]. To address the knowledge gap for the frequency of three enteric pathogens in the region, we conducted a series of experiments aimed at characterizing features of genetic diversity of these pathogens in Tibet. Molecular epidemiology data generated from the results could help in assessment of their zoonotic potential and public health significance in areas without or less intensive farming.

\section{Results \\ Cryptosporidium}

Among 577 samples (62 from Mainling, 125 from Gongbo'gyamda, 262 from Bayi District, 56 from Gyaca, and 72 from Xaitongmoin), Cryptosporidium spp. were detected in eight $(1.4 \%, 8 / 577)$ specimens based on the SSU rRNA gene. Infection rates ranged from 0 to $6.9 \%$ among the five counties (Table 1), and differences among counties were statistically significant $(P<0.05)$. The highest infection rate $(6.9 \%)$ was observed in Xaitongmoin, and no Cryptosporidium was detected in Mainling or Gyaca. The molecular analysis of eight successfully sequenced Cryptosporidium-positive products revealed the presence of two species, $C$. andersoni $(n=$ $7)$ and $C$. bovis $(n=1)$, with $C$. andersoni being predominant $(7 / 8,87.5 \%)$. All $C$. andersoni samples were detected in Bayi District and Xaitongmoin and their sequences showed 100\% homology to FJ463172 derived from dairy calves in China. C. bovis was only identified in Gongbo'gyamda, with no nucleotide difference compared with KT922231 derived from calves in Ethiopia.

The $C$. andersoni-positive samples were characterized by MLST, and four of seven samples were simultaneously amplified based on four microsatellite/minisatellite loci (MS1, MS2, MS3, and MS16). One MLST subtype (A5,A4,A2,A1) was found in the present study.

\section{Giardia duodenalis}

For G. duodenalis, ten samples $(1.7 \%, 10 / 577)$ were tested positive using the SSU rRNA and tpi genes. G. duodenalis was observed in all counties but Mainling 
Table 1 Species, assemblage, and genotype distribution of three enteric pathogens in the present study

\begin{tabular}{|c|c|c|c|c|c|c|c|}
\hline \multirow[t]{2}{*}{ Location } & \multirow{2}{*}{$\begin{array}{l}\text { Sample } \\
\text { size }\end{array}$} & \multicolumn{3}{|c|}{ Prevalence (\%) $(95 \% \mathrm{Cl})$} & \multicolumn{3}{|c|}{ Species/Assemblage/Genotype (no.) } \\
\hline & & Cryptosporidium & G. duodenalis & E. bieneusi & Cryptosporidium & G. duodenalis & E. bieneusi \\
\hline Mainling & 62 & 0 & 0 & $12.9(4.3-21.5)$ & 0 & 0 & BEB4 (8) \\
\hline Gongbo'gyamda & 125 & $0.8(0.0-2.4)$ & $2.4(0.0-5.1)$ & $1.6(0.0-3.8)$ & C. bovis (1) & E (3) & I (2) \\
\hline Bayi District & 262 & $0.8(0.0-1.8)$ & $1.5(0.0-3.0)$ & $4.6(2.0-7.1)$ & C. andersoni (2) & $E(4)$ & BEB4 (6), I (4), J (2) \\
\hline Gyaca & 56 & 0 & $3.6(0.0-8.6)$ & $5.4(0.0-11.4)$ & 0 & $E(2)$ & $\mathrm{CHC8}(1), \mathrm{D}(2)$ \\
\hline Xaitongmoin & 72 & $6.9(0.9-13.0)$ & $1.4(0.0-4.2)$ & $5.6(0.1-11.0)$ & C. andersoni (5) & $E(1)$ & D (3), BEB6 (1) \\
\hline Total & 577 & $1.4(0.4-2.3)$ & $1.7(0.7-2.8)$ & $5.0(3.2-6.8)$ & C. andersoni (7) C. bovis (1) & E (10) & BEB4 (14), I (6), D (5), J (2), CHC8 (1), BEB6 (1) \\
\hline
\end{tabular}

(Table 1), and the infection rates of G. duodenalis were 3.6\% (Gyaca), 2.4\% (Gongbo'gyamda), 1.5\% (Bayi District), and $1.4 \%$ (Xaitongmoin); rates did not differ significantly by region $(P>0.05)$. Analysis of eight SSU rRNA sequences and six tpi sequences showed that all sequences belonged to $G$. duodenalis assemblage E. At the SSU rRNA locus, all the assemblage E sequences obtained here $(n=8)$ showed $100 \%$ homology to KX259145 previously recognized from sika deer in China. By comparison with $G$. duodenalis tpi sequences available on GenBank, the sequences $(n=6)$ were all identical to isolate KY710747 derived from dairy cattle in China.

\section{Enterocytozoon bieneusi}

Among the 577 yaks examined, 29 (5.0\%, 29/577) were tested positive for $E$. bieneusi based on the ITS region. $E$. bieneusi was seen in all counties, with infection rates ranging from 1.6 to $12.9 \%$ (Table 1). Yaks in Mainling had the highest infection rate $(12.9 \%)$ whereas those in Gongbo'gyamda had the lowest (1.6\%) occurrence, and infection rates differed significantly between five counties $(P<0.05)$. All positive samples were successfully sequenced, and six previously described genotypes were identified: BEB4 $(n=14), \mathrm{I}(n=6), \mathrm{D}(n=5), \mathrm{J}(n=2)$, $\mathrm{CHC} 8(n=1)$, and BEB6 $(n=1)$. The evolutionary relationships and zoonotic potential were analyzed by Bayesian analysis of the E. bieneusi ITS genotypes. Genotype D was clustered into group 1 (high zoonotic potential) in the phylogenetic analysis. Genotypes I, J, BEB4, $\mathrm{CHC} 8$, and BEB6 were clustered into group 2 (Fig. 1). The dominant genotype BEB4 was detected in Mainling and Bayi District; genotype I was observed in Gongbo'gyamda and Bayi District; genotype D was seen in Gyaca and Xaitongmoin; and genotypes J, CHC8, and BEB6 were identified in Bayi District, Gyaca, and Xaitongmoin, respectively.

MLST analysis was conducted on the E. bieneusi-positive samples, targeting four markers (MS1, MS3, MS4, and MS7). Among the 29 specimens, 14, 9, 11, and 15 specimens were successfully amplified at MS1, MS3, MS4, and MS7, respectively. However, ITS-positive specimens from Gyaca, Gongbo'gyamda, and Xaitongmoin were all negative in amplification of MS3 locus. At the
MS4 locus, 9 of 11 sequences, successfully amplified, were obtained from Mainling and Bayi District. Altogether, sequence analysis yielded 6, 2, 2, and 3 subtypes at the MS1, MS3, MS4, and MS7 loci, respectively. Additionally, 9 samples (four from Bayi District and five from Mainling) were simultaneously positive at all four loci, forming three distinct MLGs (Table 2). The number of specimens with complete data for all four loci was limited because some specimens were not amplified at all loci, especially in the PCR analysis of the MS3 locus.

\section{Discussion}

In the present study, the overall infection rate of Cryptosporidium was $1.4 \%(8 / 577)$, which is higher than that in one report of yaks in Sichuan $(1.2 \%, 1 / 84)$ [19]. However, molecular epidemiological data have shown the prevalence here was lower than two reports in Gansu (5.3\%, 4/76 and 6.0\%, 7/117), Tibet $(9.1 \%, 4 / 44)$ and Qinghai (ranging from 3.3 to $30.0 \%$ in five studies) [10, 19-23]. Additionally, compared with dairy cattle, the distribution of Cryptosporidium species according to the yak age remains unknown [9]. Yaks were raised freely on pastures by herders under traditional and natural grazing condition; even herdsmen cannot make clear the age information of yaks sampled, so the precise age of individual yaks was not available during our sample collection. C. andersoni and C. bovis were identified in our study, which were identical to the species reported previously in Gansu [20]. C. andersoni was the dominant species in the present study, in agreement with one case in Qinghai reported by $\mathrm{Li}$ [22], whereas $C$. parvum was the most common species in the central-western region of China reported by Qi [19], and C. bovis was the dominant species in one study in Gansu reported by Qin and three reports in Qinghai [10, 20, 21, 23].

Many reports suggested that humans are frequently infected with $C$. hominis and $C$. parvum, whereas $C$. andersoni and $C$. bovis are of low zoonotic risk to humans [8]. C. bovis has been observed in human with diarrhea from India, Australia, and Egypt [24-26]. More studies are required to clarify the potential zoonotic transmission of C. andersoni and C. bovis. Additionally, 


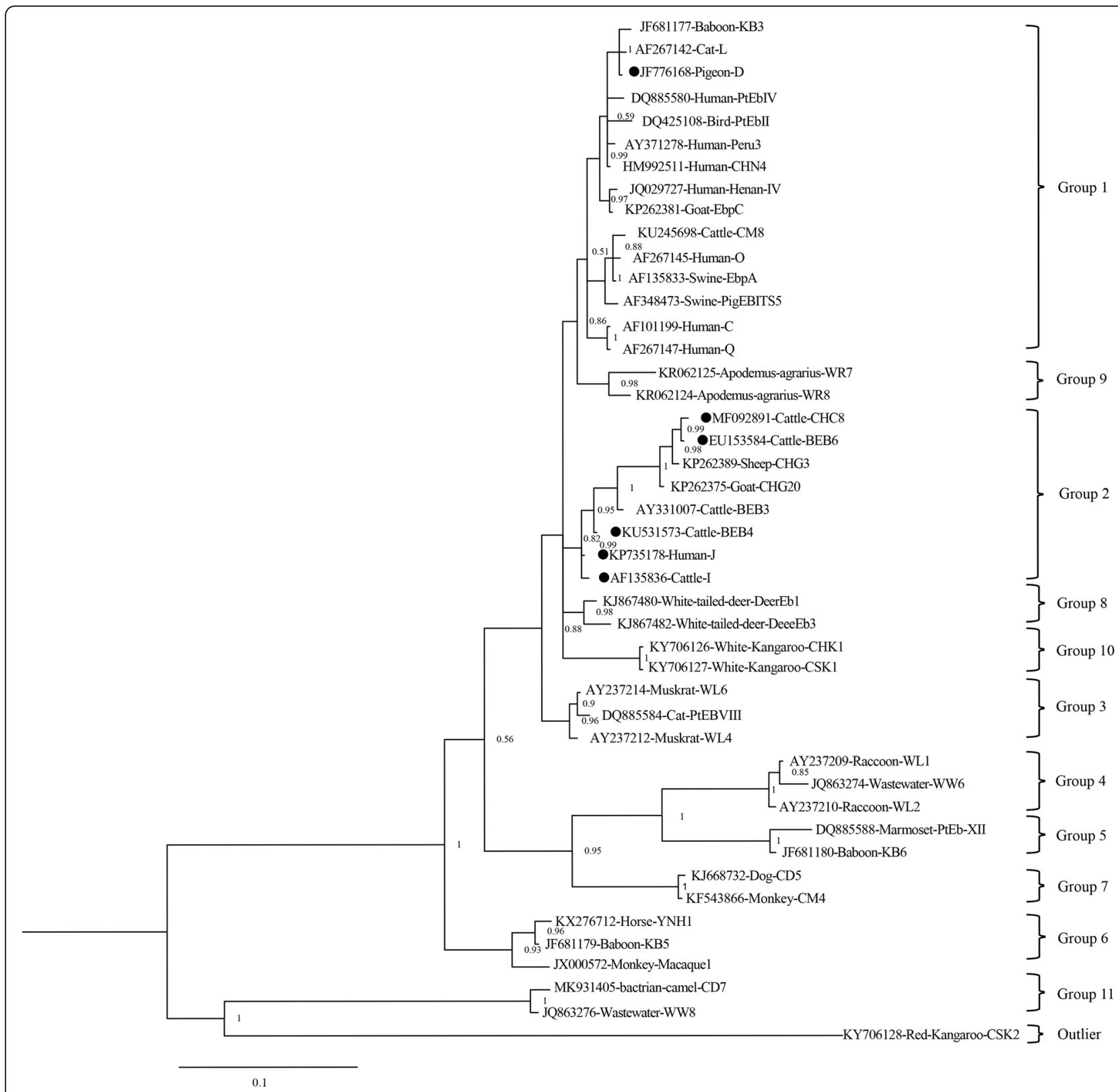

Fig. 1 Phylogenetic tree based on Bayesian analysis of the E. bieneusi ITS genotypes. Statistically significant posterior probabilities are indicated on the branches. Each sequence was labeled with its accession number, host origin, and genotype designation. CSK2 (isolated from a kangaroo in China with GenBank accession no. KY706128) was considered as outlier. Genotypes detected in this study are indicated by filled circles

the high-resolution genotyping tool MLST has been developed to characterize the population genetics and transmission of C. andersoni [27]. Over twenty MLST subtypes for $C$. andersoni have been identified in animals, most of which were isolated in dairy cattle [27-32]. Because of the limited number of isolates, four of seven $C$. andersoni-positive specimens yielded one subtype (A5,A4, $\mathrm{A} 2, \mathrm{~A} 1)$. However, the subtype $(\mathrm{A} 4, \mathrm{~A} 4, \mathrm{~A} 4, \mathrm{~A} 1)$ was reported to be most common in dairy and beef cattle in Henan [29], Shaanxi [31], and Heilongjiang [28]; dairy cattle in Guangdong [32]; and $\mathrm{He}$ cattle in Xinjiang [30], whereas the subtype (A2,A4,A2,A1) was the most prevalent subtype for dairy cattle in Xinjiang [30].

Compared with previous data obtained from other reports in yaks, the prevalence of G. duodenalis in our study $(1.7 \%, 10 / 577)$ was slightly higher than that reported in Sichuan $(1.2 \%, 1 / 36)$, and $G$. duodenalis was not detected from 44 yaks in another report from Tibet [19]. The infection rate here was lower than two reports from Gansu $(1.9 \%, 4 / 208$ and 3.4\%, 3/117) [19, 33], and Qinghai (3.3$10.4 \%$ in four reports) $[10,19,34,35]$. Differences in prevalence can be explained by some factors in the 
Table 2 Multilocus sequence typing of E. bieneusi genotypes based on minisatellite/microsatellite loci

\begin{tabular}{|c|c|c|c|c|c|c|c|}
\hline \multirow[t]{2}{*}{ Location } & \multirow{2}{*}{$\begin{array}{l}\text { Sample } \\
\text { ID }\end{array}$} & \multirow{2}{*}{$\begin{array}{l}\text { ITS } \\
\text { genotype }\end{array}$} & \multicolumn{5}{|c|}{ Multilocus genotype } \\
\hline & & & MS1 & MS3 & MS4 & MS7 & MLGs \\
\hline \multirow[t]{7}{*}{ Bayi District } & 6 & I & - & - & - & Type1 & - \\
\hline & 15 & । & Type2 & - & - & Type1 & - \\
\hline & 29 & BEB4 & Type1 & Type1 & Type1 & Type1 & MLG1 \\
\hline & 32 & BEB4 & Type2 & Type1 & Type1 & Type1 & MLG2 \\
\hline & 33 & BEB4 & Type1 & Type1 & Type1 & Type1 & MLG1 \\
\hline & 148 & । & - & - & - & Type1 & - \\
\hline & 306 & J & Type3 & Type2 & Type2 & Type 1 & MLG3 \\
\hline \multirow[t]{5}{*}{ Mainling } & 227 & BEB4 & Type2 & Type1 & Type1 & Type1 & MLG2 \\
\hline & 247 & BEB4 & Type2 & Type1 & Type1 & Type1 & MLG2 \\
\hline & 250 & BEB4 & Type2 & Type1 & Type1 & Type1 & MLG2 \\
\hline & 258 & BEB4 & Type2 & Type1 & Type1 & Type1 & MLG2 \\
\hline & 265 & BEB4 & Type2 & Type1 & Type1 & Type1 & MLG2 \\
\hline Gongbo'gyamda & 716 & । & - & - & - & Type 1 & - \\
\hline Gyaca & 772 & D & Type6 & - & - & - & - \\
\hline \multirow[t]{3}{*}{ Xaitongmoin } & 1424 & D & Type5 & - & - & - & - \\
\hline & 1435 & D & Type5 & - & Type1 & Type3 & - \\
\hline & 1439 & BEB6 & Type4 & - & Type1 & Type2 & - \\
\hline
\end{tabular}

molecular epidemiological data, including geographic separation, immunity status, sample sizes, seasons, examination methods, ecological conditions, and management systems.

Accompanied with strong host specificity, G. duodenalis assemblage $E$ is the dominant species in yaks, with only two reports of infection occurring with zoonotic assemblages A and B [12]. All positive products in the present study were in assemblage $\mathrm{E}$, which was consistent with studies in Gansu, Sichuan, and Qinghai $[19,33]$. However, two samples of assemblage A have been documented in yaks in Qinghai, and assemblage B was first detected in yaks in Qinghai [10, 34]. To date, assemblage E has been commonly identified in yaks and other domestic mammals, even though the eight assemblages are thought to have different host ranges [11]. An increasing number of documented cases of humans infected with assemblage $\mathrm{E}$ have been reported in Egypt, Brazil, and Australia [3640]. Further studies are required to clarify the reservoirs and transmission routes of G. duodenalis assemblage E.

$E$. bieneusi has been observed in a wide variety of vertebrate hosts with worldwide distribution and importance in veterinary medicine [4]. It has high prevalence and high genetic diversity in multiple provinces and municipalities in China [41]. However, few molecular epidemiology data are available to understand the occurrence and genotypes of $E$. bieneusi in Tibet. We determined the prevalence and genetic diversity of $E$. bieneusi among yaks in five districts of Tibet, and the infection rate $(5.0 \%, 29 / 577)$ was higher than previously reported in one study in Gansu $(1.1 \%, 4 / 353)$ [16], but lower than that of two studies in Qinghai (7.0\%, 40/554 and 7.2\%, 40/554) [15, 17].

Sequence analyses indicated the presence of six distinct genotypes, of which BEB4 was predominant, followed by I, D, J, CHC8, and BEB6. Phylogenetic analysis revealed that genotype $\mathrm{D}$, firstly detected in yaks, was clustered into group 1 (high zoonotic potential), and the other five genotypes belonged to group 2 (Fig. 1). The dominant genotype in our study, BEB4, was also the most common genotype in Gansu and one case in Qinghai, in which J was predominant in another report in Qinghai. Fourteen genotypes have been detected in yaks among four studies, including the present study, of which genotypes CHN11, CHN12, CHN14, WCY1, and $\mathrm{D}$ belong to group 1 and the remainder belong to group 2 [15-17]. Genotype D, observed in Gyaca and Xaitongmoin, has been commonly found in humans, nonhuman primates, and domestic and wild animals [42-49]. It is plausible that genotypes in group 2 have low zoonotic risk, yet genotypes J, BEB4, and BEB6 have been identified in humans in some studies [50-53]. Thus, the zoonotic potential and public health significance of genotypes in group 2 may be different than previously believed.

Based on the ITS locus, the current standard molecular marker for identification of E. bieneusi, the MLST technique has been applied to understand the transmission of E. bieneusi in humans and animals [54-56]. Because of the limited number of sequences obtained from the MS3 locus, only nine samples were simultaneously identified at all four loci (Table 2). Three distinct MLGs were formed, with two from genotype BEB4 and one from genotype J. 


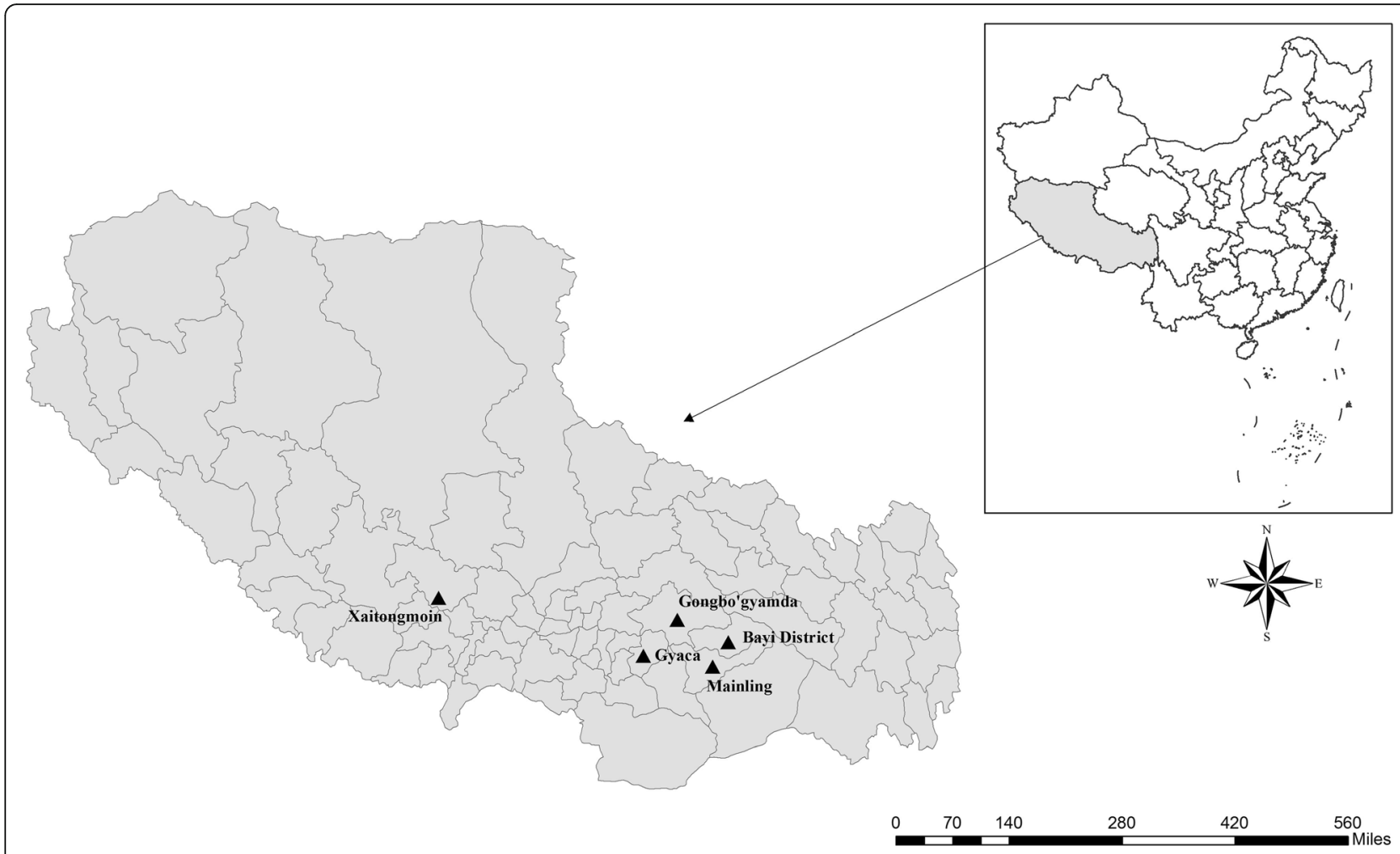

Fig. 2 Geographic map of the sampling locations in Tibet, China. The figure was originally designed by the authors under the software ArcGIS 10.2. The original vector diagram imported in ArcGIS was adapted from National Geomatics Center of China (http://www.ngcc.cn). The map has been modified and assembled according to permission and attribution guidelines

One study suggested that most herds in Tibet obtain drinking water from rivers, a water source shared among yaks and other grazing animals. There is a high risk of contamination of drinking water sources by the protozoa studied here [10]. Fresh fecal materials from bovines are used as cooking fuel by herders, further increasing the possibility of infection by these pathogens. The data on genetic diversity and phylogenetic relationships of these pathogens allow an assessment of their zoonotic potential and public health significance. Moreover, such studies in regions with a distinctive geographic environment and less intensive feeding can help us understand the distribution and transmission characteristics of Cryptosporidium, G. duodenalis, and E. bieneusi.

\section{Conclusion}

In this study, we focused on the prevalence and genetic diversity of three intestinal pathogens from yaks in Tibet. The infection rates for Cryptosporidium, G. duodenalis, and E. bieneusi were lower than those in most reports conducted in yaks of Qinghai and Gansu Provinces. One subtype for $C$. andersoni (A5,A4,A2,A1) and three MLGs for E. bieneusi (two from BEB4 and one from J) were identified using MLST. Sequence and phylogenetic analyses indicated that $C$. andersoni, $C$. bovis, G. duodenalis assemblage E, and five E. bieneusi genotypes in group 2 had a low zoonotic risk, whereas five genotype $\mathrm{D}$ sequences showed that yaks may act as a biological disseminator or mechanical vector in the transmission of E. bieneusi to humans.

\section{Methods}

Study site and sample collection

From June 2016 to July 2016, 577 fecal specimens (approximately $30 \mathrm{~g}$ each) were collected on the rangelands of Mainling, Gongbo'gyamda, and Bayi District (Nyingchi), Gyaca (Lhoka), and Xaitongmoin (Shigatse) in Tibet (Fig. 2). We sought yaks defecating, and tried our best to avoid repeated sampling. Each sample was collected from the ground immediately after defecation using a sterile disposable latex glove. To avoid fecal material that had contacted the ground, care was taken to gather only the top layer of the feces. No obvious clinical signs were observed in these yaks, except for one case of diarrhoea. The fresh samples were placed in clean plastic containers marked with relevant information, transported to the laboratory, and stored in $2.5 \%$ potassium dichromate solution at $4{ }^{\circ} \mathrm{C}$ for use in subsequent DNA extraction. 
Table 3 Primer sequences and reaction conditions used in nested PCR amplifications

\begin{tabular}{|c|c|c|c|c|}
\hline Locus & Primer sequences $\left(5^{\prime}-3^{\prime}\right)$ & Nucleotide fragment (bp) & Annealing temperature $\left({ }^{\circ} \mathrm{C}\right)$ & Reference \\
\hline \multirow[t]{4}{*}{ Cryptosporidium SSU rRNA } & SSU-F2: TTCTAGAGCTAATACATGCG & 840 & 55 & {$[57]$} \\
\hline & SSU-R2:CCCATTTCCTTCGAAACAGGA & & & \\
\hline & SSU-F3:GGAAGGGTTGTATTTATTAGATAAAG & & 55 & \\
\hline & SSU-R4:CTCATAAGG TGCTGAAGGAGTA & & & \\
\hline \multirow[t]{4}{*}{ G. duodenali SSU rRNA } & Gia2029: AAGTGTGGTGCAGACGGACTC & 292 & 55 & [58] \\
\hline & Gia2150c: CTGCTGCCGTCCTTGGATGT & & & \\
\hline & RH11: CATCCGGTCGATCCTGCC & & 59 & \\
\hline & RH4: AGTCGAACCCTGATTCTCCGCCCAGG & & & \\
\hline \multirow[t]{4}{*}{ G. duodenalis tpi } & AL3543: AAATIATGCCTGCTCGTCG & 530 & 50 & [59] \\
\hline & AL3546: CAAACCTTITCCGCAAACC & & & \\
\hline & AL3544: CCCTTCATCGGIGGTAACTT & & 50 & \\
\hline & AL3545: GTGGCCACCACICCCGTGCC & & & \\
\hline \multirow[t]{4}{*}{ E. bieneusi ITS } & EBITS3: GGTCATAGGGATGAAGAG & 389 & 57 & {$[60]$} \\
\hline & EBITS4: TTCGAGTTCTITCGCGCTC & & & \\
\hline & EBITS1: GCTCTGAATATCTATGGCT & & 55 & \\
\hline & EBITS2.4: ATCGCCGACGGATCCAAGTG & & & \\
\hline
\end{tabular}

\section{DNA extraction and PCR amplification}

To reduce the effect of potassium dichromate, each specimen was washed three times with distilled water before centrifugation at $1500 \times g$ for $10 \mathrm{~min}$ at room temperature followed by DNA extraction. According to the procedure recommended by the manufacturer, genomic DNA was extracted from $200 \mathrm{mg}$ of each specimen using the E.Z.N.A.R ${ }^{\odot}$ Stool DNA (Omega Biotek Inc., Norcross, GA, USA), and the obtained DNA was stored at $-20{ }^{\circ} \mathrm{C}$ until used in the PCR analysis.

To screen for Cryptosporidium spp., G. duodenalis, and $E$. bieneusi infection, the corresponding loci were used as described previously. Cryptosporidium was identified by the SSU rRNA gene [57]; G. duodenalis was identified based on the SSU rRNA [58] and tpi genes [59]; and E. bieneusi was detected by nested PCR targeting the partial region of ITS [60] (Table 3). With the Applied Biosystems 2720 Thermal Cycler (Applied Biosystems, Foster City, USA), the amplification for Cryptosporidium and E. bieneusi was performed in $25 \mu \mathrm{L}$ volume with $1 \mu \mathrm{L}$ extracted DNA sample, $2.5 \mu \mathrm{L} 10 \times$ KOD-Plus PCR buffer, $2.5 \mu \mathrm{L}$ dNTPs $(2 \mathrm{mM}), 1.5 \mu \mathrm{L}$ MgSO4 (25 mM), $0.5 \mu \mathrm{L}$ each primer $(25 \mu \mathrm{M}), 16 \mu \mathrm{L}$ double distilled water, and $0.5 \mu \mathrm{L}$ KOD-Plus amplification enzyme (1 unit/ $\mu \mathrm{L}$ ) (ToYoBo Co., Ltd., Osaka, Japan). The PCR reaction mixture reactions for G. duodenalis loci were conducted: $2.5 \mu \mathrm{L} 1 \times$ PCR buffer, $2 \mu \mathrm{L}$ dNTPs $(1.25 \mathrm{mM}), 0.3 \mu \mathrm{L}$ each primer $(25 \mu \mathrm{M}), 0.2 \mu \mathrm{L}$ rTaq DNA polymerase (1 unit/ $\mu \mathrm{L})$ (Takara Shuzo Co., Ltd), $2 \mu \mathrm{L}$ of DNA sample, $16.7 \mu \mathrm{L}$ double distilled water, and $1 \mu \mathrm{L}$ of bovine serum albumin $(10 \mathrm{mg} / \mathrm{mL})$. Samples positive for $C$. andersoni and E. bieneusi were selected and characterized by the multilocus sequence typing (MLST) technique, using nested PCR amplifications targeting the microsatellite/minisatellite loci (MS1, MS2, MS3, and MS16 for C. andersoni), and (MS1, MS3, MS4, and MS7 for E. bieneusi) [27, 54]. Samples that were positive at all four loci were used to deduce the MLGs of C. andersoni and E. bieneusi. The pre-existing positive DNA samples stored in lab were set for positive control. Controls were included in all PCRs runs.

The secondary PCR products were separated by $1 \%$ agarose gel electrophoresis following staining with DNA Green (TIANDZ, Beijing, China) and visualized on a UV transilluminator. The positive secondary amplification products were sequenced on an ABI PRISM ${ }^{\mathrm{Tm}} 3730 \mathrm{XL}$ DNA Analyzer using the BigDye Terminator v3.1 Cycle Sequencing Kit (Applied Biosystems, Foster City, CA, USA). Data accuracy was confirmed with two-directional sequencing.

\section{Data analysis}

To characterize the phylogenetic analysis of sequences of the ITS regions obtained here and reference sequences downloaded from GenBank. Bayesian inference (BI) and Monte Carlo Markov Chain (MCMC) methods were used to construct phylogenetic trees in MrBayes, version 3.2.6 (http://mrbayes.sourceforge.net/). Determined by ModelTest, version 3.7 (http://www.molecularevolution.org/), the general time reversible model $(\mathrm{GTR}+\mathrm{G})$ was the best-fit nucleotide substitution model. The number of substitutions (Nst) was set at six, and posterior probability values were calculated by running 1,000,000 generations with four simultaneous tree- 
building chains. Trees were saved every 1000th generation. At the end of each run, the standard deviation of the split frequencies was $<0.01$, and the potential scale reduction factor approached one. A 50\% majority rule consensus tree was constructed for each analysis using the final $75 \%$ of the trees generated via BI. FigTree, version 1.3.1 (http://tree.bio.ed.ac.uk/software/figtree/), was used to visualize and edit the maximum clade credibility by these analyses.

\section{Statistical analysis}

The infection rates of $95 \%$ confidence intervals (CI) were calculated by Wald method of SPSS 22.0 version (SPSS Inc., Chicago, IL, United States). Differences in corresponding infection rates among locations were examined by the Chi-square test, and differences were considered significant at $P<0.05$.

\section{Abbreviations}

CAFO: Concentrated Animal Feeding Operation; Cl: Confidence intervals; ITS: Internal transcribed spacer; MLST: Multilocus sequence typing; SSU rRNA: Small subunit rRNA; tpi: triosephosphate isomerase

\section{Acknowledgements}

We thank Louise Adam, ELS(D), from Liwen Bianji, Edanz Editing China (www.liwenbianji.cn/ac) for editing the English text of a draft of this manuscript.

\section{Authors' contributions}

$\mathrm{LXZ}$ contributed to the conception and design of the experiments. YYW, YKC and JKL were involved in the sample collection. YYW, YCC, YKC, XQZ, SJZ, LW, and DFL performed the experiments. YYW, FCJ, SMZ, RJW, and CSN contributed to analysis of results and data interpretation. YYW and LXZ contributed to manuscript drafting. All authors read and approved the final version of the manuscript.

\section{Funding}

The study was partially supported by the National Key Research and Development Program of China (2017YFD0501305, 2017YFD0500405), the Natural Science Foundation of China (30600603, 31672548), the Natural Science Foundation of Henan Province (162300410129), and the Chinese Agricultural Research Systems (CARS-37). The funding body provided monetary support only, and the design of the study including collection, analysis, and interpretation of data, and in writing the manuscript were not influenced by the funders.

\section{Availability of data and materials}

All data generated and/or analyzed during this study are included in this published manuscript. The 24 representative nucleotide sequences obtained have been deposited in GenBank database under the following accession numbers: MK139948 for Cryptosporidium, MK140454 to MK140456 and MK165410 for C. andersoni MLST subtype, MK139942 to MK139947 for E. bieneusi, MK140446 to MK140453 and MK165405 to MK165409 for E. bieneusi MLST subtype.

\section{Ethics approval and consent to participate}

Strictly in accordance with the Law of the People's Republic of China on the Protection of Wildlife of adopted in 1988, verbal consent to participate was obtained from all participating owners or managers of pasture and yaks in Tibet prior to our study, and all field studies complied with guidelines of local legislation. All applicable international, national and institutional guidelines for animal care and use were observed. In addition, the research protocol was reviewed and approved by the Research Ethics Committee of Henan Agricultural University. No yaks were injured during specimen collection, and no endangered or protected species were involved in the present study.

\section{Consent for publication}

Not applicable.

\section{Competing interests}

The authors declare that they have no competing interests.

\section{Author details}

${ }^{1}$ College of Animal Science and Veterinary Medicine, Henan Agricultural University, Zhengzhou 450002, Henan Province, China. ${ }^{2}$ International Joint Research Laboratory for Zoonotic Diseases of Henan, Zhengzhou 450002, Henan Province, China. ${ }^{3}$ College of Veterinary Medicine, Huazhong Agricultural University, Wuhan 430070, Hubei Province, China. ${ }^{4}$ Laboratory of Detection and Monitoring of Highland Animal Disease, Tibet Agriculture and Animal Husbandry College, Linzhi 860000, Tibet, China.

Received: 27 June 2019 Accepted: 8 November 2019

Published online: 21 November 2019

\section{References}

1. Xiao L. Molecular epidemiology of cryptosporidiosis: an update. Exp Parasitol. 2010;124(1):80-9.

2. Wang $R$, Wang $H$, Sun $Y$, Zhang L, Jian F, Qi M, Ning C, Xiao L. Characteristics of Cryptosporidium transmission in preweaned dairy cattle in Henan, China. J Clin Microbiol. 2011;49(3):1077-82

3. Feng Y, Xiao L. Zoonotic potential and molecular epidemiology of Giardia species and giardiasis. Clin Microbiol Rev. 2011;24(1):110-40.

4. Santin M, Fayer R. Microsporidiosis: Enterocytozoon bieneusi in domesticated and wild animals. Res Vet Sci. 2011;90(3):363-71.

5. Xiao L, Fayer R. Molecular characterisation of species and genotypes of Cryptosporidium and Giardia and assessment of zoonotic transmission. Int J Parasitol. 2008;38(11):1239-55.

6. Baldursson S, Karanis P. Waterborne transmission of protozoan parasites: review of worldwide outbreaks - an update 2004-2010. Water Res. 2011; 45(20):6603-14.

7. Blackburn BG, Mazurek JM, Hlavsa M, Park J, Tillapaw M, Parrish M, Salehi E, Franks W, Koch E, Smith F, Xiao L, Arrowood M, Hill V, da Silva A, Johnston $\mathrm{S}$, Jones JL. Cryptosporidiosis associated with ozonated apple cider. Emerg Infect Dis. 2006;12(4):684-6.

8. Ryan U, Fayer R, Xiao L. Cryptosporidium species in humans and animals: current understanding and research needs. Parasitology. 2014 141(13):1667-85.

9. Wang R, Zhao G, Gong Y, Zhang L. Advances and perspectives on the epidemiology of bovine Cryptosporidium in China in the past 30 years. Front Microbiol. 2017:8:1823.

10. Wang G, Wang G, Li X, Zhang X, Karanis G, Jian Y, Ma L, Karanis P. Prevalence and molecular characterization of Cryptosporidium spp. and Giardia duodenalis in 1-2-month-old highland yaks in Qinghai Province, China. Parasitol Res. 2018;117(6):1793-800.

11. Caccio SM, Ryan U. Molecular epidemiology of giardiasis. Mol Biochem Parasitol. 2008;160(2):75-80.

12. Li J, Wang H, Wang R, Zhang L. Giardia duodenalis infections in humans and other animals in China. Front Microbiol. 2017:8:2004.

13. Thellier M, Breton J. Enterocytozoon bieneusi in human and animals, focus on laboratory identification and molecular epidemiology. Parasite. 2008; 15(3):349-58.

14. Karim MR, Wang R, Dong H, Zhang L, Li J, Zhang S, Rume FI, Qi M, Jian F, Sun M, Yang G, Zou F, Ning C, Xiao L. Genetic polymorphism and zoonotic potential of Enterocytozoon bieneusi from nonhuman primates in China. Appl Environ Microbiol. 2014;80(6):1893-8.

15. Ma J, Cai J, Ma J, Feng Y, Xiao L. Enterocytozoon bieneusi genotypes in yaks (Bos grunniens) and their public health potential. J Eukaryot Microbiol. 2015; 62(1):21-5.

16. Ma J, Zhang N, Hou J, Zou Y, Hu G, Zhu X, Zhou D. Detection of Enterocytozoon bieneusi in white yaks in Gansu Province, China. Biomed Res Int. 2017;2017:5790181.

17. Zhang Q, Cai J, Li P, Wang L, Guo Y, Li C, Lei M, Feng Y, Xiao L. Enterocytozoon bieneusi genotypes in Tibetan sheep and yaks. Parasitol Res. 2018;117(3):721-7.

18. Li K, Shahzad M, Zhang H, Jiang X, Mehmood K, Zhao X, Li J. Socioeconomic burden of parasitic infections in yaks from 1984-2017 on Qinghai Tibetan plateau of China-a review. Acta Trop. 2018;183:103-9. 
19. Qi M, Cai J, Wang R, Li J, Jian F, Huang J, Zhou H, Zhang L. Molecular characterization of Cryptosporidium spp. and Giardia duodenalis from yaks in the central western region of China. BMC Microbiol. 2015;15:108.

20. Qin S, Zhang X, Zhao G, Zhou D, Yin M, Zhao Q, Zhu X. First report of Cryptosporidium spp. in white yaks in China. Parasit Vectors. 2014;7:230.

21. Mi R, Wang X, Li C, Huang Y, Zhou P, Li Z, Lei M, Cai J, Chen Z. Prevalence and genetic characterization of Cryptosporidium in yaks in Qinghai Province of China. PLoS One. 2013;8(9):e74985.

22. Li P, Cai J, Cai M, Wu W, Li C, Lei M, Xu H, Feng L, Ma J, Feng Y, Xiao L. Distribution of Cryptosporidium species in Tibetan sheep and yaks in Qinghai, China. Vet Parasitol. 2016;215:58-62.

23. Ma J, Cai J, Ma J, Feng Y, Xiao L. Occurrence and molecular characterization of Cryptosporidium spp. in yaks (Bos grunniens) in China. Vet Parasitol. 2014; 202(3-4):113-8.

24. Khan SM, Debnath C, Pramanik AK, Xiao L, Nozaki T, Ganguly S. Molecular characterization and assessment of zoonotic transmission of Cryptosporidium from dairy cattle in West Bengal, India. Vet Parasitol. 2010; 171(1-2):41-7.

25. Ng JS, Eastwood K, Walker B, Durrheim DN, Massey PD, Porigneaux P, Kemp R, McKinnon B, Laurie K, Miller D, Bramley E, Ryan U. Evidence of Cryptosporidium transmission between cattle and humans in northern New South Wales. Exp Parasitol. 2012;130(4):437-41.

26. Helmy YA, Krucken J, Nockler K, von Samson-Himmelstjerna G, Zessin $\mathrm{KH}$. Molecular epidemiology of Cryptosporidium in livestock animals and humans in the Ismailia province of Egypt. Vet Parasitol. 2013;193(1-3): $15-24$.

27. Feng Y, Yang W, Ryan U, Zhang L, Kvac M, Koudela B, Modry D, Li N, Fayer $R$, Xiao L. Development of a multilocus sequence tool for typing Cryptosporidium muris and Cryptosporidium andersoni. J Clin Microbiol. 2011; 49(1):34-41.

28. Zhao W, Wang R, Zhang W, Liu A, Cao J, Shen Y, Yang F, Zhang L. MLST subtypes and population genetic structure of Cryptosporidium andersoni from dairy cattle and beef cattle in northeastern China's Heilongjiang Province. PLoS One. 2014;9(7):e102006.

29. Wang R, Jian F, Zhang L, Ning C, Liu A, Zhao J, Feng Y, Qi M, Wang H, Lv C, Zhao G, Xiao L. Multilocus sequence subtyping and genetic structure of Cryptosporidium muris and Cryptosporidium andersoni. PLoS One. 2012;7(8):e43782.

30. Qi M, Wang R, Jing B, Jian F, Ning C, Zhang L. Prevalence and multilocus genotyping of Cryptosporidium andersoni in dairy cattle and He cattle in Xinjiang, China. Infect Genet Evol. 2016;44:313-7.

31. Zhao G, Ren W, Gao M, Bian Q, Hu B, Cong M, Lin Q, Wang R, Qi M, Qi M, Zhu X, Zhang L. Genotyping Cryptosporidium andersoni in cattle in Shaanxi Province, Northwestern China. PloS one. 2013;8(4):e60112.

32. Liang N, Wu Y, Sun M, Chang Y, Lin X, Yu L, Hu S, Zhang X, Zheng S, Cui Z, Zhang L. Molecular epidemiology of Cryptosporidium spp. in dairy cattle in Guangdong Province, South China. Parasitology. 2018;146(1):28-32.

33. Song G, Qin S, Zhao G, Zhu X, Zhou D, Song M. Molecular characterization of Giardia duodenalis from white yaks in China. Acta Parasitol. 2016;61(2):397-400.

34. Jin Y, Fei J, Cai J, Wang X, Li N, Guo Y, Feng Y, Xiao L. Multilocus genotyping of Giardia duodenalis in Tibetan sheep and yaks in Qinghai, China. Vet Parasitol. 2017;247:70-6.

35. Wang G, Wang G, Li X, Ma L, Karanis G, Christodoulou-Vafeiadou E, Karanis P. Detection of Giardia duodenalis assemblage $\mathrm{E}$ infections at the Tibetan plateau area: yaks are suitable hosts. Acta Trop. 2017;169:157-62.

36. Foronda P, Bargues MD, Abreu-Acosta N, Periago MV, Valero MA, Valladares B, Mas-Coma S. Identification of genotypes of Giardia intestinalis of human isolates in Egypt. Parasitol Res. 2008;103(5):1177-81.

37. Helmy YA, Klotz C, Wilking H, Krucken J, Nockler K, Von Samson-Himmelstjerna $\mathrm{G}$, Zessin $\mathrm{KH}$, Aebischer T. Epidemiology of Giardia duodenalis infection in ruminant livestock and children in the Ismailia province of Egypt: insights by genetic characterization. Parasit Vectors. 2014;7:321.

38. Abdel-Moein KA, Saeed H. The zoonotic potential of Giardia intestinalis assemblage $E$ in rural settings. Parasitol Res. 2016;115(8):3197-202.

39. Fantinatti M, Bello AR, Fernandes O, Da-Cruz AM. Identification of Giardia lamblia assemblage $\mathrm{E}$ in humans points to a new Anthropozoonotic cycle. J Infect Dis. 2016;214(8):1256-9.

40. Zahedi A, Field D, Ryan U. Molecular typing of Giardia duodenalis in humans in Queensland - first report of assemblage E. Parasitology. 2017;144(9):1154-61.
41. Wang S, Wang R, Fan X, Liu T, Zhang L, Zhao G. Prevalence and genotypes of Enterocytozoon bieneusi in China. Acta Trop. 2018;183:142-52.

42. Liu H, Jiang Z, Yuan Z, Yin J, Wang Z, Yu B, Zhou D, Shen Y, Cao J. Infection by and genotype characteristics of Enterocytozoon bieneusi in HIV/AIDS patients from Guangxi Zhuang autonomous region, China. BMC Infect Dis. 2017;17(1):684

43. Wang L, Zhang H, Zhao X, Zhang L, Zhang G, Guo M, Liu L, Feng Y, Xiao L. Zoonotic Cryptosporidium species and Enterocytozoon bieneusi genotypes in HIVpositive patients on antiretroviral therapy. J Clin Microbiol. 2013;51(2):557-63.

44. Yu F, Wu Y, Li T, Cao J, Wang J, Hu S, Zhu H, Zhang S, Wang R, Ning C, Zhang L. High prevalence of Enterocytozoon bieneusi zoonotic genotype D in captive golden snub-nosed monkey (Rhinopithecus roxellanae) in zoos in China. BMC Vet Res. 2017;13(1):158.

45. Karim MR, Wang R, He X, Zhang L, Li J, Rume Fl, Dong H, Qi M, Jian F, Zhang S, Sun M, Yang G, Zou F, Ning C, Xiao L. Multilocus sequence typing of Enterocytozoon bieneusi in nonhuman primates in China. Vet Parasitol. 2014;200(1-2):13-23.

46. Du S, Zhao G, Shao J, Fang Y, Tian G, Zhang L, Wang R, Wang H, Qi M, Yu S. Cryptosporidium spp., Giardia intestinalis, and Enterocytozoon bieneusi in captive non-human Primates in Qinling Mountains. Korean J Parasitol. 2015; 53(4):395-402.

47. Li J, Dong H, Wang R, Yu F, Wu Y, Chang Y, Wang C, Qi M, Zhang L. An investigation of parasitic infections and review of molecular characterization of the intestinal protozoa in nonhuman primates in China from 2009 to 2015. Int J Parasitol Parasites Wildl. 2017;6(1):8-15.

48. Qi M, Jing B, Jian F, Wang $R$, Zhang $S$, Wang $H$, Ning $C$, Zhang L. Dominance of Enterocytozoon bieneusi genotype $J$ in dairy calves in Xinjiang, Northwest China. Parasitol Int. 2017;66(1):960-3.

49. Zhao W, Zhang W, Yang F, Cao J, Liu H, Yang D, Shen Y, Liu A. High prevalence of Enterocytozoon bieneusi in asymptomatic pigs and assessment of zoonotic risk at the genotype level. Appl Environ Microbiol. 2014;80(12):3699-707.

50. Wang L, Xiao L, Duan L, Ye J, Guo Y, Guo M, Liu L, Feng Y. Concurrent infections of Giardia duodenalis, Enterocytozoon bieneusi, and Clostridium difficile in children during a cryptosporidiosis outbreak in a pediatric hospital in China. PLoS Negl Trop Dis. 2013;7(9):e2437.

51. Zhang X, Wang Z, Su Y, Liang X, Sun X, Peng S, Lu H, Jiang N, Yin J, Xiang $M$, Chen $Q$. Identification and genotyping of Enterocytozoon bieneusi in China. J Clin Microbiol. 2011;49(5):2006-8.

52. Ye J, Ji Y, Xu J, Ma K, Yang X. Zoonotic Enterocytozoon bieneusi in raw wastewater in Zhengzhou, China. Folia Parasitol (Praha). 2017;64:002.

53. Sak B, Brady D, Pelikanova M, Kvetonova D, Rost M, Kostka M, Tolarova V, Huzova Z, Kvac M. Unapparent microsporidial infection among immunocompetent humans in the Czech Republic. J Clin Microbiol. 2011; 49(3):1064-70

54. Feng Y, Li N, Dearen T, Lobo ML, Matos O, Cama V, Xiao L. Development of a multilocus sequence typing tool for high-resolution genotyping of Enterocytozoon bieneusi. Appl Environ Microbiol. 2011;77(14):4822-8.

55. Li W, Cama V, Akinbo FO, Ganguly S, Kiulia NM, Zhang X, Xiao L. Multilocus sequence typing of Enterocytozoon bieneusi: lack of geographic segregation and existence of genetically isolated sub-populations. Infect Genet Evol. 2013;14:111-9.

56. Wang X, Wang R, Ren G, Yu Z, Zhang L, Zhang S, Lu H, Peng X, Zhao G. Multilocus genotyping of Giardia duodenalis and Enterocytozoon bieneusi in dairy and native beef (Qinchuan) calves in Shaanxi province, northwestern China. Parasitol Res. 2016;115(3):1355-61.

57. Xiao L, Singh A, Limor J, Graczyk TK, Gradus S, Lal A. Molecular characterization of Cryptosporidium oocysts in samples of raw surface water and wastewater. Appl Environ Microbiol. 2001;67(3):1097-101.

58. Appelbee AJ, Frederick LM, Heitman TL, Olson ME. Prevalence and genotyping of Giardia duodenalis from beef calves in Alberta, Canada. Vet Parasitol. 2003;112(4):289-94.

59. Sulaiman IM, Fayer R, Bern C, Gilman RH, Trout JM, Schantz PM, Das P, Lal AA, Xiao L. Triosephosphate isomerase gene characterization and potential zoonotic transmission of Giardia duodenalis. Emerg Infect Dis. 2003;9(11):1444-52.

60. Buckholt MA, Lee JH, Tzipori S. Prevalence of Enterocytozoon bieneusi in swine: an 18-month survey at a slaughterhouse in Massachusetts. Appl Environ Microbiol. 2002;68(5):2595-9.

\section{Publisher's Note}

Springer Nature remains neutral with regard to jurisdictional claims in published maps and institutional affiliations. 